\title{
Brain morphology and neurological soft signs in adolescents with first-episode psychosis
}

Joost Janssen, Angeles Diaz-Caneja, Santiago Reig, Igor Bombín, María Mayoral, Mara Parellada, Montserrat Graell, Dolores Moreno, Arantzazu Zabala, Veronica García Vazquez, Manuel Desco and Celso Arango

\section{Background}

Adolescents with first-episode psychosis have increased severity of neurological soft signs when compared with controls, but it is unclear whether increased severity of neurological soft signs is an expression of specific structural brain deficits.

\begin{abstract}
Aims
To examine whether increased severity of neurological soft signs was associated with decreased brain volumes in adolescents with first-episode psychosis.

\section{Method}

Brain scans were obtained for 70 adolescents (less than 18 years of age) with first-episode psychosis (duration of positive symptoms less than 6 months). volumes were assessed using voxel-based
\end{abstract}

morphometry and through segmentation of anatomical structures.

\section{Results}

Increased severity of sensory integration neurological soft signs correlated with smaller right and left thalamus volume, whereas increased severity of sequencing of complex motor acts neurological soft signs correlated with smaller right caudate volume.

\section{Conclusions}

Neurological soft signs may be an easy-to-assess marker of region-specific structural brain deficits in adolescents with first-episode psychosis.

\section{Declaration of interest}

None.
Neurological soft signs are minor neurological abnormalities that are not believed to be part of a well-defined neurological syndrome. ${ }^{1}$ Neurological soft signs are more severe in adults and adolescents with psychosis, including schizophrenia (treated and antipsychotic-naive), ${ }^{2,3}$ first-episode psychosis ${ }^{4-6}$ and bipolar I disorder, ${ }^{7}$ compared with age-matched healthy controls. Psychosis during adolescence is associated with structural brain abnormalities. ${ }^{8}$ In adults with first-episode psychosis, decreased volumes of the basal ganglia structures, thalamus, heteromodal cortex and cerebellar grey matter have been associated with increased severity of neurological soft signs. ${ }^{9-14}$ In adolescents with psychosis, to the best of our knowledge, no study has investigated the relationship between severity of neurological soft signs and volumes of specific brain structures. The current study used voxel-based morphometry and segmentation of anatomical structures to investigate whether increased severity of neurological soft signs predicted decreased brain volumes in adolescents (younger than 18 years of age) with first-episode psychosis (duration of positive symptoms less than 6 months). Considering the complex anatomical definition of some structures related to the severity of neurological soft signs, such as the heteromodal cortex, we chose to focus only on the thalamus, caudate and putamen because the segmentation algorithms used were deemed more reliable for these structures.

\section{Method}

\section{Participants}

Individuals were recruited from the two child and adolescent psychiatric in-patient units in Madrid (Hospital General Universitario Gregorio Marañon and Hospital Universitario Infantil Niño Jesus). The two units serve a population of approximately five million people. All patients consecutively seen at these facilities between April 2002 and November 2005 who fulfilled the inclusion criteria described below were invited to participate in the study. Seventy-four individuals agreed to participate. Of the 74, 3 individuals did not undergo a magnetic resonance imaging (MRI) scan because of fear. One participant was excluded because of insufficient image quality for neuroimaging analyses, thus leaving a sample of 70 individuals (51 males). The inclusion criteria for participants were: age between 7 and 18 years, and presence of positive psychotic symptoms (within a DSM-IV ${ }^{15}$ diagnosis) for less than 6 months at the time of assessment on enrolment in the study. Exclusion criteria were: presence of a concomitant Axis I disorder, mental retardation, a pervasive developmental disorder, neurological diseases, a history of head trauma with loss of consciousness, and pregnancy. Individuals with substance use and/or dependence were excluded. Individuals with substance use were included only if positive symptoms remained present after 14 days of a negative urine screen. Prior treatment was not an exclusion criterion if positive symptoms persisted for more than 2 weeks after a negative urine drug screen. The study was approved by the institutional review boards of both participating clinical centres. After the study was thoroughly explained to all of the participants, written informed consent was obtained. All of the participants met MRI safety criteria.

\section{Clinical assessment}

All participants met DSM-IV criteria for a first episode of psychosis, assessed using the Kiddie - Schedule for Affective Disorders - Present and Lifetime Version (K-SADS-PL). ${ }^{16}$ Clinical diagnostic interviews were performed during the initial hospitalisation by four experienced psychiatrists trained in this interview. Diagnostic consensus was reached in cases where presence or absence of psychiatric diagnoses was in doubt. 
Baseline diagnoses were reassessed in a clinical interview at a 12-month follow-up visit; follow-up diagnoses were based on DSM-IV criteria. Three diagnostic categories were established: schizophrenia, $n=25$ (19 males); bipolar I disorder, $n=20$ (13 males); and 'other psychoses', $n=25$ (19 males). The latter group included six individuals with schizoaffective disorder, five with schizophreniform disorder, three with major depression with psychotic features, nine with psychosis not otherwise specified, and two with brief psychotic episode.

Psychotic symptoms were assessed using the validated Spanish version of the Positive and Negative Syndrome Scale (PANSS). ${ }^{17}$ Intraclass correlation coefficients for the four psychiatrists who administered the scale ranged from 0.72 to 0.96 . Ratings on the Global Assessment of Functioning (GAF) scale were used as a measure of overall functioning. Sociodemographic data were collected, including parental socioeconomic level as measured by the Hollingshead-Redlich scale, ${ }^{18}$ and a physical examination (e.g. weight, height) was performed.

\section{Neurological soft signs}

All participants go through a comprehensive physical examination when they are admitted to hospital as part of our first-episode clinical guidelines. The severity of neurological soft signs was evaluated using the Neurological Evaluation Scale (NES), ${ }^{19}$ a 26-item scale with four subscales: sensory integration, motor coordination, sequencing of complex motor acts, and an 'other' subscale that evaluates short-term memory, frontal release signs and eye movement abnormalities (see online supplement). Each item is rated from 0 to 2 : 0 , no abnormality; 1 , mild, but definite impairment; and 2, marked impairment. The snout and suck reflexes are scored either 0 or 2 . The severity for each subscale was calculated by summing the scores on that particular subscale. The NES was administered in its entirety, according to the original instructions of the instrument's developers, by three neuropsychologists. The intraclass correlation coefficients for the four NES subscales obtained by the examiners in an independent sample of ten individuals ranged from 0.80 to 0.99 . The neurological assessment was performed when the participants were clinically stable. The mean duration between neurological assessment and scan acquisition was 15 days $($ s.d. $=22)$.

The age at onset of psychosis was defined as the age at which the individual experienced positive psychotic symptoms for the first time. This information was gathered during the K-SADS-PL interview with parents or legal guardians. Duration of psychosis was defined as the time between age at onset of psychosis and scan acquisition. Duration of treatment was defined as the time between initiation of antipsychotic treatment at enrolment in the study and scan acquisition.

\section{Medication}

At the time of the baseline assessment, all participants were on antipsychotic medication. Seventy-six per cent $(n=53)$ of the sample $(n=70)$ were receiving only one antipsychotic, whereas $24 \%(n=17)$ were receiving two antipsychotics simultaneously. With the exception of two individuals, all participants were receiving a second-generation antipsychotic. The distribution of antipsychotic treatment was as follows: $54 \%(n=38)$ risperidone, $34 \%(n=24)$ quetiapine, 28\% $(n=20)$ olanzapine, $4 \%(n=3)$ ziprasidone, and $3 \%(n=2)$ haloperidol. The chlorpromazine equivalent dose $^{20}$ was calculated from the dose of antipsychotics received (Table 1). The mean daily antipsychotic dose in chlorpromazine equivalents was $290.0 \mathrm{mg}$ (s.d. $=180.4 \mathrm{mg}$ ).

\section{MRI acquisition}

All participants were scanned on a 1.5-T Philips MRI scanner (Philips Gyroscan, Philips Medical Systems, Best, The Netherlands). Two MR sequences were obtained for all the participants: a $\mathrm{T}_{1}$-weighted, 3-dimensional, gradient-echo scan with $1001.5-\mathrm{mm}$ contiguous axial slices (echo time (TE) $4.6 \mathrm{~ms}$; repetition time (TR) $9.3 \mathrm{~ms}$; flip angle $30^{\circ}$; field of view (FOV) $256 \mathrm{~mm}$; and in-plane voxel size $0.98 \times 0.98 \mathrm{~mm}^{2}$ ) and a $\mathrm{T}_{2}$-weighted turbo spin echo scan with $3.5-\mathrm{mm}$ contiguous axial slices (turbo factor 15; TE $=120 \mathrm{~ms}$; TR $=5809 \mathrm{~ms}$; FOV $=256 \mathrm{~mm}$; and in-plane voxel size $0.98 \times 0.98 \mathrm{~mm}^{2}$ ). Both $\mathrm{T}_{1}$ - and $\mathrm{T}_{2}$-weighted images were used for clinical neurodiagnostic evaluation by an independent neuroradiologist. No participants showed clinically significant brain pathology.

\begin{tabular}{|c|c|}
\hline & Adolescents with first-episode psychosis $(n=70)$ \\
\hline Gender, male/female: $n$ (\%) & $51 / 19(72 / 27)$ \\
\hline Handedness, right/left/ambidextrous, $n$ (\%) & $63 / 6 / 1(90 / 9 / 1)$ \\
\hline Age, years: mean (s.d.), range & $16.3(1.7), 11-18$ \\
\hline Height, cm: mean (s.d.) ${ }^{a}$ & $168.4(9.5)$ \\
\hline Weight, kg: mean (s.d.) ${ }^{a}$ & $61.3(11.8)$ \\
\hline Level of education, years: mean (s.d.) & $8.2(2.1)$ \\
\hline Parental socioeconomic status: mean (s.d.) & $2.6(1.4)$ \\
\hline Age at onset of psychosis, years: mean (s.d.), range ${ }^{a}$ & $15.3(1.8), 10-17$ \\
\hline Duration of psychosis, weeks: mean (s.d.), range ${ }^{b}$ & $12.6(10.1), 0-48$ \\
\hline Duration of treatment, weeks: mean (s.d.), range ${ }^{b}$ & $2.8(3.4), 0-25$ \\
\hline Chlorpromazine equivalents, mg: mean (s.d.), range & 290.0 (180.4), 3-920 \\
\hline Global Assessment of Functioning scale score: mean (s.d.) & $41.2(15.8)$ \\
\hline \multicolumn{2}{|l|}{ Positive and Negative Syndrome Scale score: mean (s.d.) } \\
\hline Positive symptoms & $25.3(5.6)$ \\
\hline Negative symptoms & $23.4(7.2)$ \\
\hline General psychopathology & $48.2(9.8)$ \\
\hline Cerebral grey matter, cc: mean (s.d.) & $750.4(71.8)$ \\
\hline
\end{tabular}




\section{Image processing}

\section{Voxel-based morphometry}

Voxel-based morphometry processing ${ }^{21}$ was performed using the Statistical Parametric Mapping package (SPM5; The Wellcome Department of Imaging Neuroscience, University College London). In order to improve spatial normalisation, brainextracted images were used as inputs. ${ }^{22}$ Brain-extracted images were obtained using the Brain Extraction Tool (version 2) algorithm, ${ }^{23}$ and then manually edited to remove remaining non-intracranial tissue voxels with in-house software. The locally developed software is part of a Multimodality Workstation running on IDL (Interactive Data Language, Research Systems Inc, Boulder, Colorado, USA; http://www.ittvis.com/Product Service/IDL.aspx). This software incorporates modules for triplanar visualisation, manual tracing, automatic segmentation, multimodality registration, among other advanced image processing and quantification tools. Brain-extracted images were normalised and segmented into grey matter, white matter and cerebrospinal fluid, using the a priori template segments. ${ }^{24}$ To improve segmentation accuracy, a Markov random field estimate was applied to the grey matter segmentations. All brains were visually inspected to determine whether our image processing steps resulted in successful scan normalisation and segmentation. Registration with the template was consistent across the cohort in all brain regions.

Prior to entering the grey matter segmentations in the statistical analyses, the grey matter segmentations were modulated by the Jacobian determinants from the spatial normalisation. Modulation was done to account for the contraction and expansion of grey matter during normalisation. ${ }^{25}$ After modulation, the voxel values represent grey matter volume. Finally, the modulated grey matter segmentations were smoothed using a $10-\mathrm{mm}$ Gaussian kernel to ensure that the data were sufficiently normal to justify the application of Gaussian random field theory in the statistical calculations. ${ }^{26}$

\section{Segmentation of anatomical structures}

The segmentation of anatomical structures was done using the Fmrib Integrated Registration and Segmentation Tool (FIRST) and the raw (not brain-extracted) $\mathrm{T}_{1}$-weighted images. ${ }^{27}$ The FIRST is an automatic surface-based segmentation tool that relies on trained models of shape and intensity. It utilises a multivariate Gaussian model of shape (vertex coordinates). Shapes are fitted to new data by maximising the joint probability of shape and intensity, where the search space is limited to the principal components of the vertices. The sample used to train FIRST was composed of 317 manually segmented $\mathrm{T}_{1}$-weighted MR images, with an age range from approximately 4 to 87 years. The manual segmentations in the training data were based on previously described neuroanatomical criteria for manual segmentation. ${ }^{28}$ The training data were composed of data on healthy controls as well as individuals diagnosed with schizophrenia and bipolar disorder. All segmentations were visually inspected to determine whether the segmentation process was successful.

\section{Data analysis}

Data were analysed in a two-step design. In step one, using voxelbased morphometry and small volume correction masks of the thalamus, caudate and putamen from the Wake-Forest University Pick Atlas, ${ }^{29}$ the association between the voxel-based volume in the three subcortical structures and the NES subscale scores (the NES subscale score represented the severity of neurological soft signs on that subscale) was examined. In step two, findings from step one were complemented by segmentation of those subcortical structures containing voxels that were significantly associated with NES subscale scores. After segmentation, the whole subcortical structure volume was calculated. Thereafter, the association between whole subcortical structure volume and NES subscale scores was examined.

\section{Regression models: covariates}

All analyses (in steps one and two) testing the association between voxel-based and whole subcortical structure volumes with the NES subscale scores were done using multiple regression in the context of the general linear model.

Age, gender and medication are known to influence severity of neurological soft signs as well as brain morphology. ${ }^{4,30}$ In addition, controlling for whole brain grey matter volume is advisable in voxel-based morphometry ${ }^{31}$ to avoid the influence of inter-individual differences in whole brain grey matter volume when investigating local grey matter volume changes. Therefore we entered age, gender, whole brain grey matter volume and chlorpromazine equivalent dose as covariates in all analyses (in steps one and two).

The PANSS negative symptom score was also included as a covariate, because it was significantly positively associated with the sensory integration subscale score $(P=0.001)$, the sequencing of complex motor acts subscale score $(P=0.02)$ and the 'other' signs subscale score $(P=0.01)$. An association between negative symptom severity (as measured by the PANSS) and severity of neurological soft signs has been previously reported in adolescent psychosis. ${ }^{5}$ Moreover, this association has been replicated in numerous prior studies. ${ }^{32,33}$ It has been suggested that this association indicates that negative symptoms have a stronger neuropathological basis compared with positive symptoms. Following this rationale, an association between brain morphology and severity of neurological soft signs might be interpreted as an outcome of the underlying relationship between severity of negative symptoms and neurological soft signs. Hence, the PANSS negative symptom score was included as a covariate to ensure that the association between volumes and neurological soft signs remained significant regardless of its potential correlation with negative symptoms.

\section{Step one}

Four regression models, one for each NES subscale, tested whether voxel-based volume in the three subcortical structures could be predicted from the NES subscale score. First, a whole brain grey matter $t$-map was generated. The $t$-map was then thresholded at $3.23(P<0.001)$. Thereafter, the small volume correction was applied. Within each small volume correction mask, a group of contiguous voxels (i.e. a cluster) of any size, containing one or more voxels with $P<0.05$, corrected for multiple comparisons at the voxel-level using the statistical parametric mapping familywise error, ${ }^{34}$ was considered significant. To facilitate interpretation, the coordinates of voxels with a corrected $P<0.05$ ('local maxima') were converted from normalised space to Talairach and Tournoux space, ${ }^{35}$ using the icbm2tal transform. ${ }^{36}$

\section{Step two}

After segmentation, regression models tested whether whole subcortical structure volume could be predicted from the NES subscale score. To examine the specificity of our findings, the volume of the hippocampus, which was assumed not to have a relationship with NES subscale scores, ${ }^{4}$ was also estimated using FIRST. In all analyses, to ensure robustness of the findings, outliers (if any) were removed and analyses were re-done. 


\section{Results}

The demographic and clinical characteristics of the participants are shown in Table 1 and the mean NES subscale scores are shown in Table 2. For the four voxel-based morphometry multiple regression analyses, significant findings were found for the sensory integration subscale and the sequencing of complex motor acts subscale.

\section{Sensory integration signs}

A cluster comprising the left and right side of the anterior part of the thalamus showed a significant negative association with sensory integration subscale score, meaning that increased severity of sensory integration signs was associated with smaller volume in the anterior region of the thalamus after adjusting for age, gender, whole brain grey matter volume, negative symptom score and chlorpromazine equivalent dose (Talairach coordinates of the local maxima $x, y, z:-3,-5,13 \mathrm{~mm}$; $t$-test $=-4.1$, cluster size 813 voxels; Fig. 1(a) and online Fig. DS1). Following the significant cluster located in the thalamus, the thalamus was segmented. Mean total (left + right) thalamus volume was $13.1 \mathrm{cc}$ $($ s.d. $=1.5)$. There was a significant negative association between total thalamus volume and sensory integration subscale score, similar to the voxel-based morphometry analysis, (unstandardised regression coefficient, $b=-0.15 \mathrm{cc}$ (s.e.) $=0.06, t$-test $=-2.32$,

\begin{tabular}{|c|c|c|c|}
\hline \multirow[b]{2}{*}{ Neurological Evaluation Scale subscale scores } & \multicolumn{3}{|c|}{ Adolescents with first-episode psychosis $(n=70)$} \\
\hline & Mean & s.d. & Range \\
\hline Sensory integration ${ }^{a}$ & 4.9 & 2.3 & $0-11$ \\
\hline Motor coordination & 3.8 & 2.1 & $0-11$ \\
\hline Motor sequencing & 4.9 & 4.1 & $0-25$ \\
\hline Other & 11.1 & 4.1 & $1-20$ \\
\hline
\end{tabular}

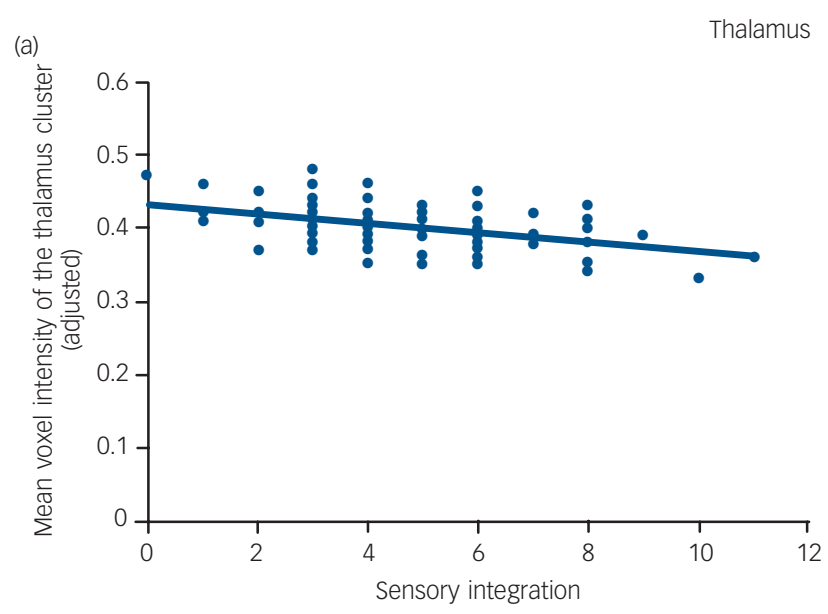

(c)

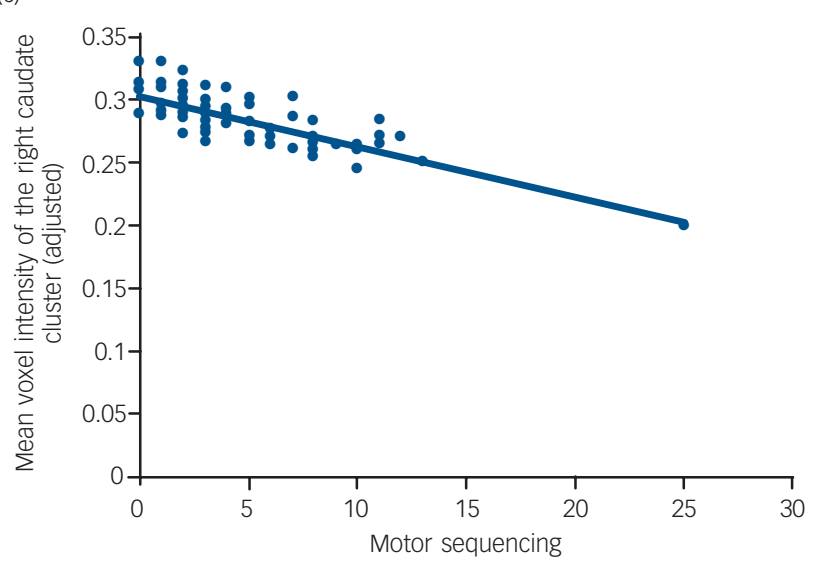

(b)

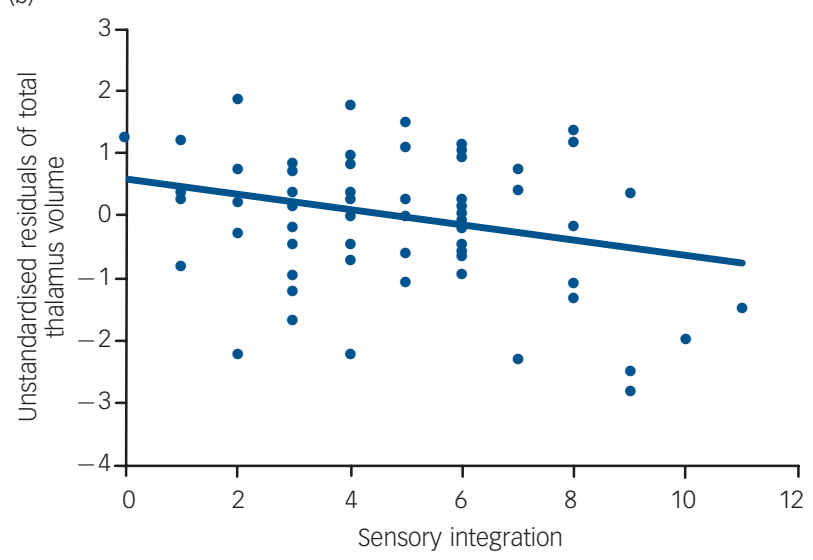

(d)

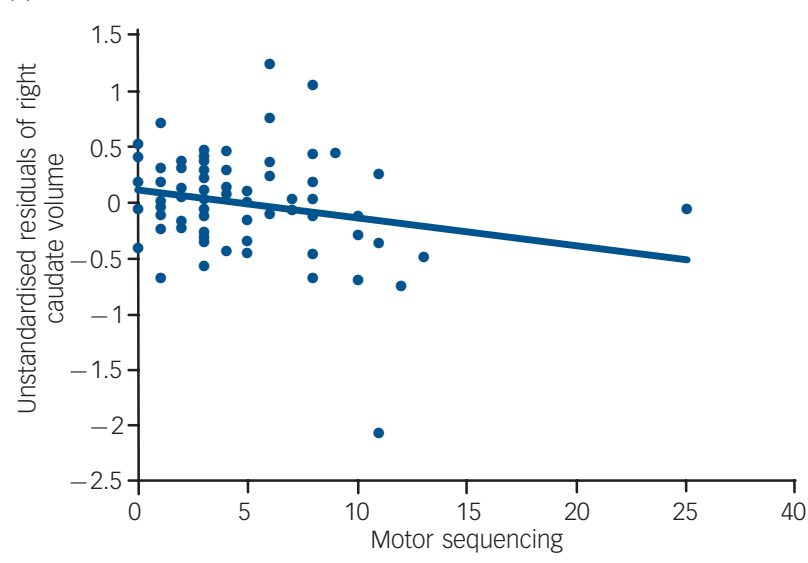

Fig. 1 Plots showing the association between severity of sensory integration and sequencing of complex motor acts scores and thalamus and right caudate volume in 70 adolescents with first-episode psychosis.

Left column shows voxel-based morphometry data, right column shows volume data after segmentation. Upper row: (a) the significant association, for every participant, between sensory integration subscale score and the mean voxel-intensity values of the cluster located in the thalamus and (b) the significant association between the sensory integration subscale score and the unstandardised residuals of total thalamus volume. Values are adjusted for age, gender, whole brain grey matter volume, negative symptom score and antipsychotic dose in chlorpromazine equivalents. Bottom row: similar data for the sequencing of complex motor acts subscale score and right caudate volume (c, d). 
degrees of freedom (d.f.) $=62, \quad P=0.02$ ), representing a $1 \%$ decrease in total thalamus volume per increment in sensory integration subscale score, after correction for age, gender, whole brain grey matter volume, negative symptom score and chlorpromazine equivalent dose (Fig. 1(b)).

\section{Sequencing of complex motor acts}

A cluster comprising the right caudate showed a significant negative association with sequencing of complex motor acts subscale score, meaning that increased severity of neurological soft signs on the sequencing of complex motor acts subscale was associated with smaller volume in this region (Talairach coordinates of the local maxima $x, y, z: 13,-9,20 \mathrm{~mm} ; t$ test $=-4.0$; cluster size 529 voxels, small volume correction corrected $P<0.05$; Fig. 1 (c) and online Fig. DS1).

Subsequently, the right caudate was segmented. Mean right caudate volume was $2.4 \mathrm{cc}$ (s.d.=0.5). A negative relationship was found between right caudate volume and sequencing of complex motor acts subscale score $(b=-0.03 c c$ (s.e. $=0.02)$, $t$-test $=-2.1$, d.f. $=63, P=0.04)$, representing a $1 \%$ decrease in right caudate volume per increment in sequencing of complex motor acts subscale score, after correction for age, gender, whole brain grey matter volume, negative symptom score and chlorpromazine equivalent dose (Fig. 1(d)).

The outliers (motor sequencing subscale scores 11 and 25) did not change the results.

No significant associations were found between total hippocampus volume and sensory integration subscale score $(P=0.24)$ or sequencing of complex motor acts subscale score $(P=0.32)$. A post hoc exploratory analysis did not reveal any differences in severity of neurological soft signs or subcortical brain volumes between the diagnostic groups, suggesting that the relationship between neurological soft signs and brain volumes is similar for the three diagnostic groups.

\section{Discussion}

The major finding of this study is the localisation of specific neuroanatomical correlates of severity of neurological soft signs in adolescents with first-episode psychosis.

\section{Sensory integration signs: thalamus}

Increased severity of sensory integration signs was associated with decreased volume in an anterior part of the thalamus (i.e. after voxel-based morphometry analysis) and decreased total thalamus volume (i.e. after segmentation of the thalamus). Our results extend findings from a previous voxel-based morphometry study ${ }^{11}$ by showing the negative association between severity of sensory integration signs and total thalamus volume in younger people with psychosis with a considerably shorter duration of illness (12.6 weeks $v$. 70.8 weeks). The thalamus functions as a central relay station of the brain by filtering and gating sensory inputs to the cerebral cortex. ${ }^{37}$ Sensory integration functions, such as audiovisual integration and stereognosis, require transfer of sensory information from subcortical regions to multimodal cortical brain areas. Our results may indicate that structural impairment of the thalamus affects this transfer and consequently decreases performance on the sensory integration subscale. Thalamic volume decrease has been associated with chronic schizophrenia, ${ }^{38}$ first-episode psychosis $^{39}$ and increased genetic predisposition to schizophrenia. ${ }^{40}$ These findings, together with evidence of functional and post-mortem thalamic abnormalities, ${ }^{41}$ suggest that thalamic structure and function might be affected in psychosis. $^{42}$

\section{Sequencing of complex motor tasks: right caudate}

Increased severity of neurological soft signs on the sequencing of complex motor acts subscale was associated with volume decreases in the right caudate. Right and left caudate volume decreases have been associated with increased minor motor and cognitive neurological abnormalities, as measured by the NES, in adults with first-episode psychosis. ${ }^{12}$ The caudate receives cortical projections from motor areas in the frontal lobe, and the received information is passed on to the putamen, globus pallidus and substantia nigra, which together with the caudate form the basal ganglia motor system. ${ }^{43}$ Interestingly, decreased putamen volume and increased severity of neurological soft signs on the motor coordination subscale has been reported in adults with firstepisode psychosis. ${ }^{11}$ Therefore, a volume decrease in the caudate may point to suboptimal functioning of the basal ganglia motor system in people with first-episode psychosis.

No significant differences in caudate volume between firstepisode psychosis or minimally treated individuals and healthy controls have been reported. ${ }^{44}$ In people with chronic schizophrenia, enlarged caudate volumes have been reported, possibly as a result of treatment with first-generation but not secondgeneration antipsychotics. ${ }^{45}$ In the current study, we cannot rule out effects of antipsychotics on brain morphology; however, we studied a sample of adolescents with first-episode psychosis with a mean duration of treatment of less than 3 weeks. We thus deem it unlikely that treatment with primarily second-generation antipsychotics affected our results. Indeed, we included chlorpromazine equivalent dose as a covariate in our analyses and still found an association between severity of sequencing of complex motor acts and right caudate volume.

\section{Brain morphology and clinical diagnosis}

Schizophrenia and bipolar disorder overlap in clinical symptoms and in genetic and epidemiological risk factors. ${ }^{46}$ In previous MRI studies of adolescents with psychotic bipolar disorder or schizophrenia, grey matter deficits have been found in medial frontal areas in both patient groups. ${ }^{47}$ Furthermore, these frontal cortical regions have been associated with increased genetic susceptibility to adult-onset schizophrenia and bipolar I disorder, ${ }^{48}$ suggesting that both disorders share underlying neuropathology. The results from MRI studies in early-onset bipolar disorder investigating subcortical structures such as the thalamus and basal ganglia are conflicting. ${ }^{49,50}$

Few studies have examined the relationship between severity of neurological soft signs and brain morphology in people with first-episode psychosis. Our study has extended the sparse previous findings to a younger patient sample with a shorter duration of psychosis. Our findings thus point to an association between increased severity of neurological soft signs and volume decreases in the thalamus and the right caudate in adolescents with recent-onset first-episode psychosis. If replicated, these findings may be of interest for clinicians as assessment of neurological soft signs in these individuals may serve as an easyto-obtain marker of structure-specific brain alterations.

\section{Limitations}

Our results should be interpreted in view of the limitations of this study. First, the clinical heterogeneity and small sample size of diagnostic subgroups can be considered a limitation and should be taken into account when extrapolating our findings to different 
psychotic disorders. However, the current sample allows for the study of a phenotypic marker of psychosis, which is the trait that all participants have in common. Severity of neurological soft signs as well as subcortical brain volumes were not different between diagnostic groups, which suggests a shared marker for psychosis rather than one specific to schizophrenia or bipolar I disorder. This may also have relevance for future diagnostic criteria (e.g. DSM-V) as some biological markers may be consistent with a dimensional approach rather than a marker for a specific diagnosis. Second, we used voxel-based morphometry to assess the relationship between voxel-based grey matter volumes and severity of neurological soft signs. The effect size of the associations that we found is rather small and our results should be interpreted with caution, considering the limitations of MRI processing. Specifically, methodological issues regarding voxelbased morphometry have been discussed in the literature. ${ }^{51}$ Third, for methodological reasons we did not formulate an a priori hypothesis about the heteromodal cortex and cerebellar grey matter, although they have been related to severity of neurological soft signs. ${ }^{11-13}$

\section{Future directions}

Future longitudinal studies should combine follow-up assessments of neurological soft signs and neuroimaging to investigate whether neurodevelopmental changes in various neuroanatomic structures, including the heteromodal cortex and cerebellum, correlate with neurological soft signs changes and whether this association is diagnosis-specific. Our group is currently conducting such studies.

Joost Janssen, PhD, Department of Experimental Surgery, Department of Psychiatry, Adolescent Unit Hospital General Universitario Gregorio Marañón (AU-HGUGM), Madrid, and Centro de Investigación Biomédica en Red de Salud Mental (CIBERSAM); Angeles Diaz-Caneja, MD, AU-HGUGM and CIBERSAM; Santiago Reig, PhD, Department of Experimental Surgery and CIBERSAM; Igor Bombín, PhD, María Mayoral MS, Mara Parellada, MD, PhD, Montserrat Graell, MD, Dolores Mayoral, MS, Mara Parellada, MD, PhD, Montserrat Graell, MD, Dolo Moreno, MD, PhD, AU-HGUGM and CIBERSAM; Arantzazu Zabala, PhD,
AU-HGUGM, Department of Neuroscience University of the Basque Country (UPV-EHU) and CIBERSAM; Veronica García Vazquez, MS, Manuel Desco, MD, $\mathrm{PhD}$, Department of Experimental Surgery and CIBERSAM; Celso Arango, MD, PhD AU-HGUGM and CIBERSAM, Spain.

Correspondence: Joost Janssen, PhD, Hospital General Universitario Gregorio Marañon, Department of Experimental Medicine and Psychiatry, Adolescent Unit, C/ Dr. Esquerdo, 46, 28007 Madrid, Spain. Email: jjanssen@mce.hggm.es

First received 18 Mar 2008, final revision 26 Feb 2009, accepted 4 Mar 2009

\section{Funding}

This study is supported by CIBER07/09, CIBER de Salud Mental (CIBERSAM), the Spanish Ministry of Health, Instituto de Salud Carlos III, 'Fondo de Investigación Sanitaria' G03/ 032, CIBER CB06/01/0079, CDTEAM Programa CÉNIT, the Spanish Ministry of Industry and Programa Juan de la Cierva of the Spanish Ministry of Education and Science.

\section{Acknowledgements}

The authors thank the participants and their families.

\section{References}

1 Heinrichs DW, Buchanan RW. Significance and meaning of neurological signs in schizophrenia. Am J Psychiatry 1988; 145: 11-8.

2 Arango C, Bartko JJ, Gold JM, Buchanan RW. Prediction of neuropsychological performance by neurological signs in schizophrenia. Am J Psychiatry 1999; 156: $1349-57$

3 Karp BI, Garvey M, Jacobsen LK, Frazier JA, Hamburger SD, Bedwell JS, et al. Abnormal neurologic maturation in adolescents with early-onset schizophrenia. Am J Psychiatry 2001; 158: 118-22.
4 Bombin I, Arango C, Buchanan RW. Significance and meaning of neurological signs in schizophrenia: two decades later. Schizophr Bull 2005; 31: 962-77.

5 Mayoral M, Bombín I, Zabala A, Robles O, Moreno DM, Parellada M, et al. Neurological soft signs in adolescents with first episode psychosis: two-year follow up. Psychiatry Res 2008; 161: 344-8.

6 Zabala A, Robles O, Parellada M, Moreno DM, Ruiz-Sancho A, Burdalo M, et al. Neurological soft signs in adolescents with first episode psychosis. Eur Psychiatry 2006; 21: 283-7.

7 Negash A, Kebede D, Alem A, Melaku Z, Deyessa N, Shibire T, et al. Neurological soft signs in bipolar I disorder patients. J Affect Disord 2004; 80: 221-30.

8 Arango C, Kahn R. Progressive brain changes in schizophrenia. Schizophr Bull 2008; 34: 310-1.

9 Bottmer C, Bachmann S, Pantel J, Essig M, Amann M, Schad LR, et al. Reduced cerebellar volume and neurological soft signs in first-episode schizophrenia. Psychiatry Res 2005; 140: 239-50.

10 Dazzan P, Morgan KD, Chitnis X, Suckling J, Morgan C, Fearon P, et al. The structural brain correlates of neurological soft signs in healthy individuals. Cereb Cortex 2006; 16: 1225-31.

11 Dazzan P, Morgan KD, Orr KG, Hutchinson G, Chitnis X, Suckling J, et al. The structural brain correlates of neurological soft signs in AESOP first-episode psychoses study. Brain 2004; 127: 143-53.

12 Keshavan MS, Sanders RD, Sweeney JA, Diwadkar VA, Goldstein G, Pettegrew JW, et al. Diagnostic specificity and neuroanatomical validity of neurological abnormalities in first-episode psychoses. Am J Psychiatry 2003; 160: $1298-304$

13 Thomann PA, Wustenberg T, Santos VD, Bachmann S, Essig M, Schroder J. Neurological soft signs and brain morphology in first-episode schizophrenia. Psychol Med 2009; 39: 371-9.

14 Venkatasubramanian G, Jayakumar PN, Gangadhar BN, Keshavan MS. Neuroanatomical correlates of neurological soft signs in antipsychotic-naive schizophrenia. Psychiatry Res 2008; 164: 215-22.

15 American Psychiatric Association. Diagnostic and Statistical Manual of Mental Disorder (4th edn) (DSM-IV). APA, 1994.

16 Kaufman J, Birmaher B, Brent D, Rao U, Flynn C, Moreci P, et al. Schedule for Affective Disorders and Schizophrenia for School-Age Children-Present and Lifetime Version (K-SADS-PL): initial reliability and validity data. J Am Acad Child Adolesc Psychiatry 1997; 36: 980-8.

17 Peralta V, Cuesta MJ. Psychometric properties of the positive and negative syndrome scale (PANSS) in schizophrenia. Psychiatry Res 1994; 53: 31-40.

18 Hollingshead A, Redlich F. Social Class and Mental Illness: A Community Study. John Wiley, 1958.

19 Buchanan RW, Heinrichs DW. The Neurological Evaluation Scale (NES): a structured instrument for the assessment of neurological signs in schizophrenia. Psychiatry Res 1989; 27: 335-50.

20 Woods Sw. Chlorpromazine equivalent doses for the newer atypical antipsychotics. J Clin Psychiatry 2003; 64: 663-7.

21 Ashburner J, Friston KJ. Voxel-based morphometry - the methods. Neuroimage 2000; 11: 805-21.

22 Fein G, Landman B, Tran H, Barakos J, Moon K, Di Sclafani V, et al. Statistical parametric mapping of brain morphology: sensitivity is dramatically increased by using brain-extracted images as inputs. Neuroimage 2006; 30 : 1187-95.

23 Smith SM. Fast robust automated brain extraction. Hum Brain Mapp 2002 17: 143-55.

24 Ashburner J, Friston KJ. Unified segmentation. Neuroimage 2005; 26: 839-51.

25 Good CD, Johnsrude IS, Ashburner J, Henson RN, Friston KJ, Frackowiak RS. A voxel-based morphometric study of ageing in 465 normal adult human brains. Neuroimage 2001; 14: 21-36.

26 Salmond $\mathrm{CH}$, Ashburner J, Vargha-Khadem F, Connelly A, Gadian DG, Friston KJ. Distributional assumptions in voxel-based morphometry. Neuroimage 2002; 17: 1027-30.

27 Smith SM, Jenkinson M, Woolrich MW, Beckmann CF, Behrens TE, Johansen-Berg $\mathrm{H}$, et al. Advances in functional and structural MR image analysis and implementation as FSL. Neuroimage 2004; 23 (suppl 1): S208-19.

28 Frazier JA, Chiu S, Breeze JL, Makris N, Lange N, Kennedy DN, et al. Structural brain magnetic resonance imaging of limbic and thalamic volumes in pediatric bipolar disorder. Am J Psychiatry 2005; 162: 1256-65.

29 Maldjian JA, Laurienti PJ, Kraft RA, Burdette JH. An automated method for neuroanatomic and cytoarchitectonic atlas-based interrogation of fMRI data sets. Neuroimage 2003; 19: 1233-9.

30 Giedd JN. Structural magnetic resonance imaging of the adolescent brain. Ann N Y Acad Sci 2004; 1021: 77-85. 
31 Ridgway GR, Henley SM, Rohrer JD, Scahill RI, Warren JD, Fox NC. Ten simple rules for reporting voxel-based morphometry studies. Neuroimage 2008; 40 : 1429-35.

32 Compton MT, Bollini AM, McKenzie Mack L, Kryda AD, Rutland J, Weiss PS, et al. Neurological soft signs and minor physical anomalies in patients with schizophrenia and related disorders, their first-degree biological relatives, and non-psychiatric controls. Schizophr Res 2007; 94: 64-73.

33 Whitty P, Clarke M, McTigue O, Browne S, Gervin M, Kamali M, et al. Diagnostic specificity and predictors of neurological soft signs in schizophrenia, bipolar disorder and other psychoses over the first 4 years of illness. Schizophr Res 2006; 86: 110-7.

34 Worsley KJ, Marret S, Neelin P, Vandal AC, Friston KJ, Evans A. A unified statistical approach for determining significant signals in images of cerebral activation. Hum Brain Mapp 1996; 4: 58-73.

35 Talairach J, Tournoux P. Co-planar Stereotaxic Atlas of the Human Brain. Thieme Medical, 1988.

36 Lancaster JL, Tordesillas-Gutierrez D, Martinez M, Salinas F, Evans A, Zilles K, et al. Bias between $\mathrm{MNI}$ and Talairach coordinates analyzed using the ICBM-152 brain template. Hum Brain Mapp 2007; 28: 1194-205.

37 Jones EG. The Thalamus. Plenum, 1985

38 Konick LC, Friedman L. Meta-analysis of thalamic size in schizophrenia Biol Psychiatry 2001; 49: 28-38.

39 Jayakumar PN, Venkatasubramanian G, Gangadhar BN, Janakiramaiah N, Keshavan MS. Optimized voxel-based morphometry of gray matter volume in first-episode, antipsychotic-naive schizophrenia. Prog Neuropsychopharmacol Biol Psychiatry 2005; 29: 587-91.

40 Lawrie SM, Whalley HC, Abukmeil SS, Kestelman JN, Donnelly L, Miller P, et al. Brain structure, genetic liability, and psychotic symptoms in subjects at high risk of developing schizophrenia. Biol Psychiatry 2001; 49: 811-23.

41 Watis L, Chen SH, Chua HC, Chong SA, Sim K. Glutamatergic abnormalities of the thalamus in schizophrenia: a systematic review. J Neural Transm 2008; 115: $493-511$
42 Andreasen NC, O'Leary DS, Flaum M, Nopoulos P, Watkins GL, Boles Ponto $\mathrm{LL}$, et al. Hypofrontality in schizophrenia: distributed dysfunctional circuits in neuroleptic-naive patients. Lancet 1997; 349: 1730-4.

43 Alexander GE, Crutcher MD. Functional architecture of basal ganglia circuits: neural substrates of parallel processing. Trends Neurosci 1990; 13: 266-71.

44 Glenthoj A, Glenthoj BY, Mackeprang T, Pagsberg AK, Hemmingsen RP Jernigan $\mathrm{TL}$, et al. Basal ganglia volumes in drug-naive first-episode schizophrenia patients before and after short-term treatment with either a typical or an atypical antipsychotic drug. Psychiatry Res 2007; 154: 199-208.

45 Corson PW, Nopoulos P, Miller DD, Arndt S, Andreasen NC. Change in basal ganglia volume over 2 years in patients with schizophrenia: typical versus atypical neuroleptics. Am J Psychiatry 1999; 156: 1200-4.

46 Thaker G. Psychosis endophenotypes in schizophrenia and bipolar disorder. Schizophr Bull 2008; 34: 720-1.

47 Janssen J, Reig S, Parellada M, Moreno D, Graell M, Fraguas D, et al. Regional gray matter volume deficits in adolescents with first-episode psychosis. J Am Acad Child Adolesc Psychiatry 2008; 47: 1311-20.

48 McDonald C, Bullmore ET, Sham PC, Chitnis X, Wickham H, Bramon E, et al. Association of genetic risks for schizophrenia and bipolar disorder with specific and generic brain structural endophenotypes. Arch Gen Psychiatry 2004; 61: 974-84.

49 Dasari M, Friedman L, Jesberger J, Stuve TA, Findling RL, Swales TP, et al. A magnetic resonance imaging study of thalamic area in adolescent patients with either schizophrenia or bipolar disorder as compared to healthy controls. Psychiatry Res 1999; 91: 155-62.

50 Wilke M, Kowatch RA, DelBello MP, Mills NP, Holland SK. Voxel-based morphometry in adolescents with bipolar disorder: first results. Psychiatry Res 2004; 131: 57-69.

51 Bookstein FL. 'Voxel-based morphometry' should not be used with imperfectly registered images. Neuroimage 2001; 14: 1454-62.

SATRA 